\title{
Immunopathological abnormalities in the normal skin of patients with rheumatoid arthritis in relation to clinical and serological findings: a one year follow up study
}

\author{
M-L WESTEDT, ${ }^{1}$ B J VERMEER, ${ }^{2}$ C J L M MEIJER, ${ }^{+}$M R DAHA, ${ }^{3}$ \\ W M BALDWIN III, ${ }^{3}$ AND A CATS 1
}

From the Departments of ${ }^{I}$ Rheumatology, ${ }^{2}$ Dermatology, and ${ }^{3}$ Nephrology, University Hospital, Leiden; and the ${ }^{4}$ Department of Pathology, Free University, Amsterdam, The Netherlands

SUMMARY Fifty two patients with seropositive rheumatoid arthritis (RA) were studied over a period of one year to investigate possible relationships among changes of circulating immune complexes (CIC), deposits of immunoglobulins and complement around the cutaneous blood vessels, clinical activity of the disease, and the presence of extra-articular manifestations (EAM). The presence or absence of IgM and C3 in and around the cutaneous blood vessels correlated significantly with the presence or absence of extra-articular features in cross sectional and longitudinal studies. Patients with evidence of these cutaneous immune deposits also had a greater prevalence of $\mathrm{CIC}$ as determined by the $\mathrm{Clq}$ binding assay (C1qBA) or polyethylene glycol (PEG) assay for IC containing IgM (IgM IC). Although the degree of perivascular mononuclear cell infiltration around the blood vessels in the papillary dermis was related to the patients' clinical state at the initial assessment, it did not correlate with the later changes in the activity of the joint disease or the occurrence of EAM. Thus the deposition of immunoglobulin or complement, or both, seems to be independent of cellular infiltration. The meaning of these cellular infiltrates is not yet fully understood. Our study has shown that many patients with RA who appeared to have only joint disease in fact had subclinical systemic disease as reflected by a positive skin biopsy or CIC. Moreover, the disappearance of IgM deposits from the skin correlated with the disappearance of EAM and improvement of joint disease.

Key words: perivascular immune deposits, immune complexes, perivascular mononuclear cell infiltrates.

The presence of immunoglobulin (IgM) and complement components in and around the cutaneous blood vessels of the clinically uninvolved skin of patients with RA has frequently been reported. ${ }^{1-5}$ These deposits have been found to correlate with a higher prevalence of extra-articular manifestations (EAM), especially rheumatoid vasculitis (RV), ${ }^{2.35}$ and with increased disease activity. ${ }^{6}$ Raised levels of circulating immune complexes (CIC) are also associated with an active disease process and with the

Accepted for publication 1 September 1986.

Correspondence to Dr M-L Westedt, Department of Rheumatology, CQ2, Building I, C2-q, University Hospital, PO Box 9600 , 2300 RC Leiden, The Netherlands. presence of EAM in patients with seropositive RA. ${ }^{78}$

Patients with RV have significantly higher levels of CIC than do patients without clinical signs of vasculitis. ${ }^{9}$ It is possible that the IC present in patients without clinically manifest vasculitis cause subclinical damage to dermal blood vessels that is insufficient to produce vasculitis. This hypothesis is supported by the finding of perivascular deposits of immunoglobulins in clinically uninvolved skin of seropositive RA patients with CIC, many of whom also show perivascular lymphocytic infiltrates. ${ }^{6}$ Furthermore, the injection of histamine into the skin of seropositive RA patients with detectable IC can produce overt vasculitis. ${ }^{10}$ In an attempt to find 
out whether the presence of these vascular abnormalities (IgM deposition and perivascular lymphocytic infiltrate) has clinical relevance we followed up 52 patients with seropositive RA for one year. Uninvolved skin of the upper arm was biopsied at entry to the study and at the end of the study year. The patients were also given a physical examination for scoring of joint activity and the presence of EAM and, at the time of the biopsies, blood was drawn for determination of IC and for other laboratory tests.

\section{Patients and methods}

Fifty two patients with definite or classical seropositive $\mathrm{RA}^{11}$ (mean age 51 years, range $18-73$ years) were followed up for one year. Informed consent was obtained from all patients.

In 30 of these patients the duration of the disease was less than one year. They entered the study before of just after therapy with a second line drug (either an antimalarial agent, gold, or Dpenicillamine) was started. Fifteen patients, designated as established RA, had a disease duration of more than two years, 12 of them were already receiving disease modifying drugs (antimalarial drugs, gold, or D-penicillamine). One patient was given an antimalarial drug after the first assessment and two patients were given only non-steroidal anti-inflammatory drugs (NSAIDs). Seven patients had leucocytoclastic vasculitis in venules or small arteries, or both, as established in tissue sections of biopsy material (skin or muscle). Four of these latter patients were treated with corticosteroids (prednisone $10 \mathrm{mg} /$ day) combined with cytostatics (two patients azathioprine $2 \mathrm{mg} / \mathrm{kg} /$ day and one patient cyclophosphamide) and one patient received corticosteroids (prednisone $5 \mathrm{mg} /$ day) combined with Dpenicillamine. The other two patients with vasculitis received only NSAIDs.

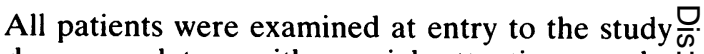
and a year later, with special attention to the presence of EAM. Joint activity was scored according to the Ritchie articular index. ${ }^{12}$ Active jointo disease was considered to be present when the Ritchie articular index score was higher than $15 . \frac{\bar{p}}{\overline{\frac{D}{a}}}$ Serum samples were obtained and a biopsy speci- $\stackrel{\mathbb{D}}{\complement}$ men of clinically uninvolved skin of the upper arm was taken on the day of the physical examination. IC were detected by the ${ }^{125} \mathrm{I}-\mathrm{Clq}$ binding assay? $(\mathrm{C} 1 \mathrm{qBA})^{13}$ and a polyethylene glycol test detecting $\vec{\omega}$ IC containing IgM (IgM IC). ${ }^{14}$

The lower normal limit for $\mathrm{C} 1 \mathrm{gBA}$ was consi-@ dered to be $20 \mu \mathrm{g}$ eq $\mathrm{AIgG} / \mathrm{ml}$. One part of the biopsy specimen of the skin was snap frozen in liquid $\vec{\sigma}$ nitrogen, cut into $4-6 \mu \mathrm{m}$ thick sections at $-20^{\circ} \mathrm{C}$, $\omega$ and stained with monospecific fluorescein $\vec{\omega}$ isothiocyanate conjugated rabbit antisera to human 0 IgG (Nordic Immunological Laboratories, Tilburg, The Netherlands), IgM, IgA, or C3 (Dakopatts, $\bar{z}$ Copenhagen, Denmark) according to established methods. ${ }^{95}$ The other part of the biopsy specimen $\stackrel{\rho}{\triangle}$ was fixed in $10 \%$ formaldehyde, and the sections $\vec{\varphi}$ were stained with haematoxylin and eosin for $@$ scoring the infiltration of mononuclear cells into the dermal vessels as described eslewhere. ${ }^{6} 15$ The observer evaluated the biopsy results without knowledge of the clinical state of the patient.

\section{Results}

Of the total group of 52 patients, 19 showed deposition of IgM or C3, or both, in and around the blood vessels in the papillary dermis both at the start? and after one year of observation (Table 1). Of@ these 19 patients, eight had an 'early' disease? process, seven had established RA, and four 3 patients had an active and recurrent vasculitis despite treatment.

Fifteen other patients showed IgM or C3 deposits,

Table 1 Signs of deposition of IgM or C3, or both, in skin biopsy specimens in relation to extra-articular manifestations (EAM) in 52 patients with $R A$ during a follow up period of one year

\begin{tabular}{|c|c|c|c|c|c|c|c|c|}
\hline \multirow{2}{*}{$\begin{array}{l}\text { Immuno- } \\
\text { fluores- } \\
\text { cence }\end{array}$} & \multirow{2}{*}{$\begin{array}{l}\text { No } \\
\text { of } \\
\text { patients }\end{array}$} & \multicolumn{3}{|c|}{ Patients with: } & \multicolumn{4}{|l|}{$E A M$} \\
\hline & & Early $R A$ & $\begin{array}{l}\text { Established } \\
R A\end{array}$ & $R V$ & $+1+*$ & $+1-$ & $-1+$ & $-1-$ \\
\hline$+/+^{*}$ & 19 & 8 & 7 & 4 & 15 & 1 & 1 & 2 \\
\hline$+1-$ & 15 & 11 & 1 & 3 & 1 & 14 & - & - \\
\hline$-1+$ & 2 & 2 & - & - & - & - & 1 & 1 \\
\hline$-1-$ & 16 & 9 & 7 & - & 3 & - & 1 & 12 \\
\hline Total & 52 & 30) & 15 & 7 & 19 & 15 & 3 & 15 \\
\hline
\end{tabular}

${ }^{*}$ Start/end of the study; $+=$ present; $-=$ absent.

Association between the presence of IgM or complement, or both. and AEM: $p<0.001$ ( $\chi^{2}$ test). 
Table 2 Signs of deposition of IgM or C3, or both, in skin biopsy specimens in relation to the articular disease activity in 52 patients with $R A$ during a follow up period of one year

\begin{tabular}{|c|c|c|c|c|c|}
\hline \multirow{2}{*}{$\begin{array}{l}\text { Immuno- } \\
\text { fluorescence }\end{array}$} & \multirow{2}{*}{$\begin{array}{l}\text { No of } \\
\text { patients }\end{array}$} & \multicolumn{4}{|c|}{ Activity* } \\
\hline & & $+1+t$ & $+1-$ & $-1+$ & $-1-$ \\
\hline$+1+\dagger$ & 19 & 7 & 5 & 4 & 3 \\
\hline$+1-$ & 15 & 2 & 9 & - & 4 \\
\hline$-1+$ & 2 & - & - & 1 & 1 \\
\hline$-1-$ & 16 & 2 & 5 & 1 & 8 \\
\hline Total & 52 & 11 & 19 & 6 & 16 \\
\hline
\end{tabular}

$*+=$ Ritchie index $>15,-=$ Ritchie index $\leqslant 15$.

†Start/end of study.

Association between the prescence of IgM or $\mathrm{C} 3$, or both, and disease activity: $p<0.05\left(\chi^{2}\right.$ test $)$.

or both, in the skin at the initial assessment but none a year later. Of these 15 patients, 11 had 'early' disease, one had well established RA, and three had $\mathrm{RV}$. In these last three patients, who had received corticosteroids and cytostatics, the signs of vasculitis had disappeared. Two of the 52 patients showed no signs of deposition of IgM or C3 at the start of the study, but such deposits were present a year later. These patients belonged to the 'early' RA group. Sixteen patients were negative initially and still negative after a year; nine of these patients belonged to the 'early' disease group and seven had established RA.

A strong positive association was found for all patients between the presence of EAM and the presence of deposits of IgM and C3 in and around the blood vessels at both assessments (Table 1) and changes in these two parameters were usually in parallel $(p<0.001)$. For example, of the 19 patients showing positive immunoglobulin deposition in and around the blood vessels both at the beginning and at the end of the study, 15 also showed persistent EAM. Of the 15 patients showing positive immunoglobulin deposition only at the start of the study, there were 14 patients in whom the EAM disappeared after one year, etc. A less significant association was seen between changes in the activity of the joint disease and changes in the deposits $(\mathrm{p}<0.05)$ (Table 2).

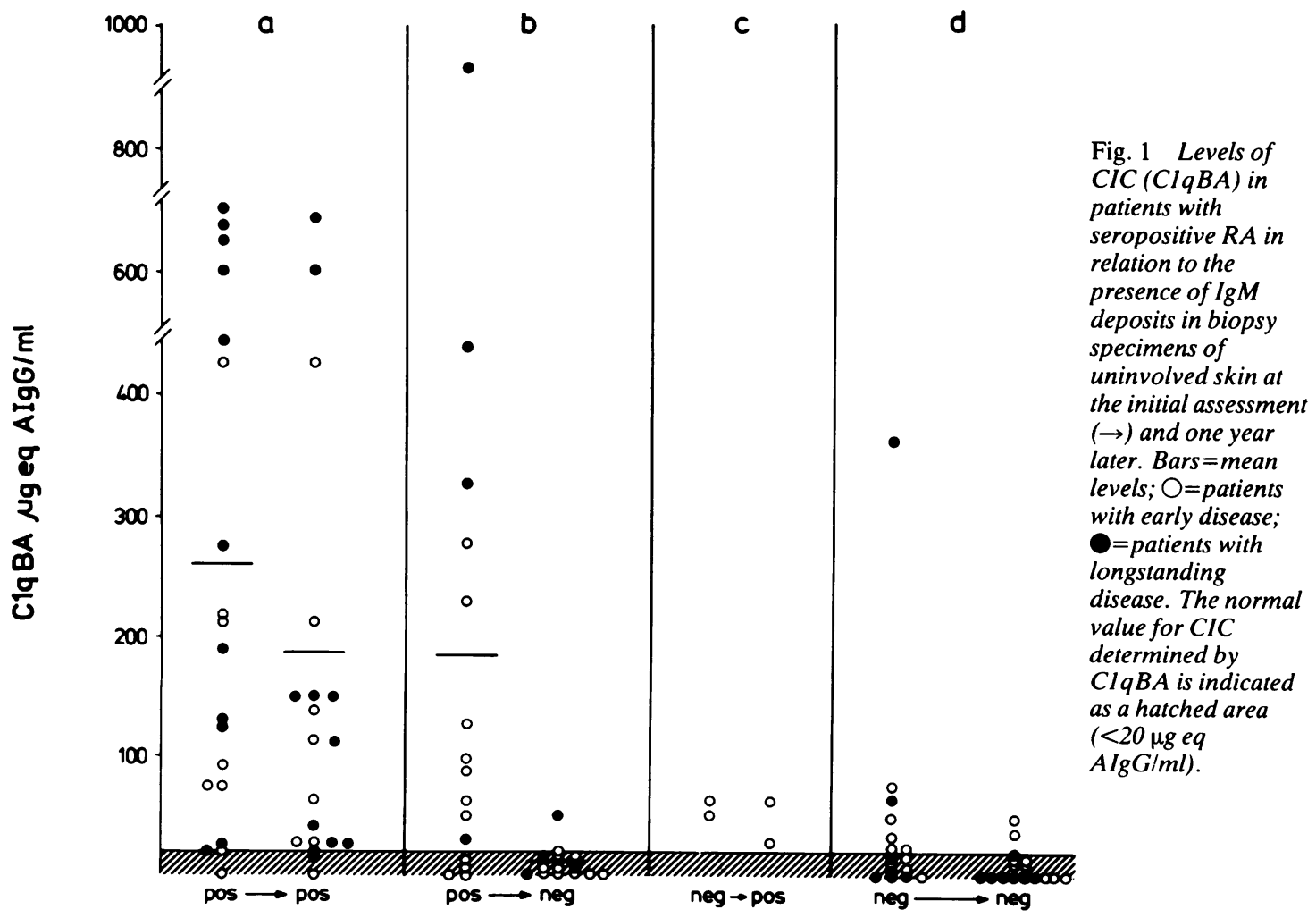

Immunofluorescence 
The presence of IC assayed with the C1qBA showed correlation with the presence of deposits in the dermal blood vessels (Fig. 1). Of the 19 patients with IgM or $\mathrm{C} 3$ deposits, or both, in both skin biopsy specimens, 18 had circulating IC detected by the C1qBA at the initial evaluation (Fig. 1a) and 17 were still positive in this respect at the second evaluation. Of the 15 patients whose biopsy specimens converted from positive immunofluorescence (IF) to negative IF, 11 had circulating IC at the initial assessment and two also at the second, whereas 13 patients showed neither IF nor IC at the second evaluation (Fig. 1b). Two patients who initially lacked but later showed immunoglobulin deposits in the skin had low levels of IC on both occasions (Fig. 1c), but the 16 patients with no detectable IgM or C3 at both assessments had significantly lower levels of circulating IC compared with patients with immunoglobulin deposits around the vessels. Similar associations were found for the IC containing IgM. Although fewer patients had circulating IgM IC when IgM and $\mathrm{C} 3$ deposits were found in the skin biopsy specimen, those with negative $\mathrm{C} 1 \mathrm{qBA}$ results and positive skin biopsies nevertheless had IC containing IgM in the circulation. Thus when IgM and $\mathrm{C} 3$ were demonstrated in and around the dermal blood vessels, IC were always detectable by the C1qBA or IgM PEG assay, or both. The reverse did not always hold true: positive IC tests were not always accompanied by demonstrable amounts of IgM and $\mathrm{C} 3$ in the skin.

Deposits of $\operatorname{IgG}$ or $\operatorname{IgA}$ were rarely found in the non-involved skin biopsy specimen from these RA patients. When present, the intensity of fluorescence was very weak and limited to only a few vessels.

Perivascular mononuclear cell infiltrates were found in 36 of the 52 patients $(69 \%)$ at the initial observation and at the second biopsy in 36 out of 50 patients $(72 \%)$ (for the other two patients the second formalin fixed biopsy specimen was not available). As reported earlier, ${ }^{6}$ the degree of

Table 3 Changes in joint activity in relation to changes in infiltrate score

\begin{tabular}{lccl}
\hline Activity & \multicolumn{3}{l}{ Infiltrate score } \\
\cline { 2 - 4 } & Increase & Decrease & No change \\
\hline Increase (RI $\uparrow \geqslant 15)^{*}$ & 3 & 0 & 3 \\
Decrease (RI $\downarrow<15)^{\dagger}$ & 0 & 11 & 7 \\
No change & 10 & 7 & 9
\end{tabular}

${ }^{*}$ Joint activity increased from inactive to active disease. †oint activity decreased from active to inactive disease. $\mathrm{RI}=$ Ritchie index . mononuclear cell infiltration was related to the patient's clinical status at the initial assessment. No $\Rightarrow$ correlation was found between changes in the score $\stackrel{5}{?}$ for infiltrate at the second biopsy and either joint? activity or the presence of IgM or C3 deposits (Table흘 $3)$. In 14 patients the infiltrate score changed in the $\frac{\bar{\rho}}{\vec{Q}}$ same direction as the disease activity, and nine $\stackrel{\mathbb{Q}}{\varrho}$ patients showed no change in either activity or@ score. Seventeen patients with RA showed no $\rightarrow$ change in clinical status but the infiltrate score changed (an increase in 10 and a decrease in seven), $\vec{\omega}$ whereas in 10 the score was unaltered but the disease activity changed from active to inactive $\frac{\mathscr{N}}{2}$ $(n=7)$ or from inactive to active $(n=3)$ (Table 3$)$.

In addition, no significant correlation was found? when the individual values for joint activity were analysed in relation to the corresponding infiltrate $\vec{\omega}$ score.

\section{Discussion}

The results of the present longitudinal study confirm our earlier findings and those of others showing $\vec{\bullet}$ immune components (mainly IgM or C3, or both) in and around blood vessels of the clinically uninvolved skin of patients with RA. ${ }^{1-6}$ In our patients theo deposition of immunoglobulin or complement, or both, in the dermal blood vessels was not onlyo associated with EAM at the first observation butक also ran parallel with the occurrence of EAM and? circulating IC with time. These changes were mosto prominent in the patients with 'early' RA, probably because they had just started treatment with antiphlogistic and remission inducing drugs. The same associations were, however, seen in the group of patients with vasculitis, who were given a more aggressive form of therapy to control the vasculitis at the beginning of the study. Correlations between the presence of perivascular immunoglobulin de-3 posits and clinical features have been found by several authors, though the frequency and the association with specific kinds of EAM have varieb widely $(25-70 \%)$ between studies. ${ }^{1-6}$ In our patientsthis variation may have been related to the severity of the disease or to the large number of patients with an early disease process.

The finding that circulating IC (C1qBA or ICW containing IgM, or both) showed correlation witk the presence of complement or IgM deposits, of both, provides indirect evidence that rheumatoide factor (RF) containing IC may be involved in the pathogenesis of damage to small blood vessels since human RF can react with heterologous immune्ष complexes in vivo and contribute to inflammation in experimental immune vasculitis. ${ }^{16}$ This hypothesis too is supported by the finding that vasculitis an 
deposition of IgM in the vessel walls can be elicited by intradermal injection of histamine into seropositive RA patients with circulating IC demonstrated by the $\mathrm{ClqBA}^{10}$ but not in RA patients without detectable circulating IC and RF.

The perivascular infiltration of mononuclear cells found in the upper dermal area in uninvolved skin, which has also been observed by others, ${ }^{4}{ }^{17}$ showed only a statistically non-significant tendency to correspond with disease articular activity. The mechanism responsible for the appearance of these infiltrates is not known, but could be a response to chemotactic factors released by cell degeneration or a reaction to deposition of immunoreactants in the vessel walls. Immunohistological stains showed that most of the mononuclear cells in the initial biopsy specimens bore the pan-T-lymphocyte marker (Leu 1) and only a few were monocytes.

$T$ cells are able to enter inflammatory sites in the skin much more easily than B cells. ${ }^{\text {is A similar }}$ subclinical vasculopathy has been found in renal transplant recipients with an active cytomegalovirus infection. In these patients the vasculopathy was correlated with RF production and circulating IC containing IgM (Baldwin, manuscript submitted). Furthermore, it is possible that in the last mentioned study the lymphocytic infiltrate appeared after the deposition of globulins and reflected a secondary event.

The present findings raise the question as to whether rheumatoid disease is a continuous spectrum with systemic manifestation occurring in a few patients or represents a composite of separate diseases. Our study has shown that many patients who appear to have only joint disease can in fact have a concurrent subclinical systemic disease. In particular in the early cases it was confirmed by 'laboratory signs' of systemic disease, i.e., a positive skin biopsy and the presence of CIC, but in most of these patients clinical manifestations remained absent during the year of the study. Furthermore, CIC which correlated with the activity of the joint disease ${ }^{19}$ were present in some patients who had not developed clinically manifest EAM during a longitudinal study lasting two years. If RA is a continuous spectrum, why do only some of the patients develop clinical vasculitis or systemic disease? The localisation of immune complexes can be influenced by many factors; for example, the composition of the CIC may differ between patients with prominent vasculitis and those without clinical vasculitis. ${ }^{19}$

In studies on mononuclear cells from patients with active $\mathrm{RV}$ the $\mathrm{C} 3 \mathrm{~b}$ and $\mathrm{Fc}$ receptors showed a defective ability to ingest and degrade soluble complexes of IgG in vitro. ${ }^{23}$ Some patients with active joint disease and raised levels of CIC had similarly defective mononuclear cells. ${ }^{23}$ There is also some evidence indicating that immunogenetic factors can regulate the occurrence of certain aspects of systemic disease in patients with RA. ${ }^{2(1-22}$

More studies must be performed before it can be determined whether a group of RA patients with a high risk of developing clinical vasculitis can be defined prospectively by characteristics such as repeated demonstration of immune deposits in the papillary vessels of uninvolved skin, defective degradation of IC by mononuclear cells in vitro, and a HLA-DR4 phenotype. If such defịnition proves possible, the institution of a more aggressive therapy at the onset of the disease might be warranted in these patients.

The study was supported by a research grant from the Dutch League against Rheumatism. The authors wish to thank Mrs $\mathrm{H} \mathrm{H}$ de Rooij-Dijk and Mrs J M Luger-Burgers for technical assistance. Mr Th Stijnen for statistical advice. and Mrs A A J P Postma for the preparation of the manuscript.

\section{References}

1 Conn D L. Schroeter A L. McDuffic F C. Cutaneous vessel immune deposits in rheumatoid arthritis. Arthritis Rheum 1976; 19: $15-20$.

2 Rapaport R J. Kozin F. Machel S E. Jordan R E. Cutaneous vascular immunofluorescence in rheumatoid arthrtitis. Correlation with circulating immune complexes and vasculitis. $A m J$ Med 1980: 68: 325-81.

3 Deicher H. Mielke H. Daniel W. et al. Studies on the vasculopathy of rheumatoid arthritis and its relation to 'systemic rheumatoid disease'. In: Deicher H. Schultz L C. eds. Arthritis: models and mechanisms. New York: Springer, 1981: 118-34.

4 Nelson H M. Mackic R M. Studies in cutancous immunofluorescence. Histological and direct immunofluorescence observations in clinically normal skin of patients with rheumatoid arthritis. J Cutan Pathol 1981: 8: 105-10.

5 McGill P E. Brougham P A. Tulloch J. Immune deposits in the skin of patients with rheumatoid arthritis. J Rheumatol 1984: 11: 105-10.

6 Westedt M-L. Meijer C J L M. Vermeer B J. Cats A. De Vries E. Rheumatoid arthritis. The clinical significance of histo- and immunopathological abnormalitics in normal skin. J Rheumatol 1984: 11: 448-53.

7 Nydegger U E. Zubler R H. Gabay R. et al. Circulating complement breakdown products in patients with rheumatoid arthritis: correlation between plasma $\mathrm{C} 3 \mathrm{~d}$, circulating immune complexes and clinical activity. J Clin Invest 1977; 59. 862-8.

8 Mumford P A. Horsfall A C. Maini R N. The frequency of circulating immune complexes in rheumatoid arthritis and systemic lupus erythematosus: a comparative study using three immuno-assays. Rheumatol Int 1982: 1: 181-6.

9 Westedt M-L. Daha M R. De Vries E, Valentijn R M. Cats A. IgA containing immune complexes in rheumatoid vasculitis and in active rheumatoid disease. $J$ Rheumatol 1985: 12: 449-55.

10 Jorizzo J L. Danicls J C. Apisarnthanarax P. Gonzalez E B. Cavallo $T$. Histamine-triggered localized vasculitis in patients with seropositive rheumatoid arthritis. J Am Acad Dermatol 1983: 9: 845-51

11 Ropes M W. Bennet G A. Cobb S. Jacox R. Jessar R A. Revision of diagnostic criteria for rheumatoid arthritis. Bull Rheum Dis 1958: 9: 175-6. 
12 Ritchic D M, Boyle J A. McInnes J M, et al. Clinical studies with an articular index for the assessment of joint tenderness in paticnts with RA. $Q J$ Med 1968; 37: 393-8.

13 Kauffmann R H. Van Es L A. Daha M R. Aggregated human immunoglobulins $G$ stabilized by albumin: a standard for immune complex detection. J Immunol Methods 1979; 31: 11-22.

14 Baldwin W M, Van Es A, Valentijn R M, Van Gemert G W. Daha M R. Van Es L A. Increased IgM and IgM complex-like material in the circulation of renal transplant recipients with primary cytomegalovirus infection. Clin Exp Immunol 1982; 50: 515-25.

15 Herrmann W A, Kauffmann R H, Van Es LA, Daha M R. Meijer C J L M. Allergic vasculitis. Arch Dermatol Res 1980; 269: $179-87$

16 Floyd M, Tesar J T. The role of $\operatorname{lgM}$ rheumatoid factor in experimental immune vasculitis. Clin Exp Immunol 1979; 36: 167-74.

17 Gonzalez T, Garcia-Montclongo R. Gantes M. Cutaneous micro vasculopathy in rheumatoid arthritis. J Rheumatol 1985; 12: 12() $6-7$.
18 Van Dinther-Janssen A C H M. Van Maarsseveen A C M Th, 心 De Groot J, Scheper R J. Comparative migration of T- and Blymphocyte subpopulations into skin inflammatory sites. Immunology 1983; 48: 518-26.

19 Westedt M-L, Daha M R. Baldwin W M. Stijnen Th, Cats A. Serum immune complexes containing IgA appear to predict crosive arthritis in a longitudinal study in rheumatoid arthritis. Ann Rheum Dis 1986; 45: 809-15.

20 Ollier W, Venables P J W, Mumford P A. et al. HLA-antigen associations with extra-articular rheumatoid arthritis. Tissue $\stackrel{\Omega}{\infty}$ Antigens 1984; 24: 279-91.

21 Westedt M-L, Breedveld F C. Schreuder G M Th. D Amaro J Cats A. De Vries R R P. Immunogenetic heterogeneity of rheumatoid arthritis. Ann Rheum Dis 1986: 45: 534-8.

22 Scott D G J, Bacon P A. Tribe C B. Systemic rheumatoid vasculitis: a clinical and laboratory study of 50 cases. Medicine $\frac{\bar{\sigma}}{2}$ (Baltimore) 1981; 60: 288-97.

23 Daha M R, Westedt M-L. Bos B, Krol H C, Van Es L A. Cats oै A. Diminished uptake and degradation of soluble complexes of $\omega$ $\operatorname{lgG}(\mathrm{AlgG})$ by monocytes of patients with rheumatoid arthritis iv and vasculitis. $\mathrm{Br} J$ Rheumatol 1986: 25: 128. 